\title{
Leveraging Global Influenza Surveillance and Response System for the COVID-19 Pandemic Response and Beyond
}

\author{
Aspen Hammond ${ }^{1, *}$; Vanessa Cozza ${ }^{1}$; Siddhi Hirve ${ }^{1}$; Marie-Jo Medina ${ }^{1}$; Dmitriy Pereyaslov ${ }^{1}$; Wenqing Zhang ${ }^{1}$
}

\section{INTRODUCTION}

Since the beginning of the coronavirus disease 2019 (COVID-19) pandemic, existing influenza surveillance systems, platforms, and capacities have been leveraged for severe acute respiratory syndrome coronavirus 2 (SARS-CoV-2) virus detection, reporting, risk assessment, and sharing of virus materials and data. This article reviews the contributions made and addresses some of the future challenges and plans for the Global Influenza Surveillance and Response System (GISRS) and associated influenza surveillance systems.

\section{DESCRIPTION}

Since 1952, the world has benefited from an international collaboration of laboratories, public health institutions, and other partners to protect people from influenza. Beginning with 26 laboratories, the World Health Organization (WHO) Global Influenza Surveillance Network (GISN), renamed as the "Global Influenza Surveillance and Response System (GISRS)" in 2011, has expanded in number and scope to now 158 institutions in 127 countries. The network has adapted to the needs and changing capacities in countries as well as events and scientific advancements, with trust and country ownership key to its success $(1-2)$.

\section{SCOPE}

From the early years of the network, there has been an emphasis on the sharing of influenza virus specimens and related information from Member States within the network and on the development and dissemination of technical guidance and standardized protocols. Member States continue to regularly share virus materials, alongside virologic and epidemiologic influenza-related information year-round. Through web-based data platforms such as FluNet (3) and FluID (4), there has been a steady increase in the number of samples tested for influenza since 2011. Since 2015, surveillance of respiratory syncytial virus (RSV) (5) based on GISRS has been piloted for integration into the influenza surveillance system. WHO, through the Global Influenza Programme (6), regularly updates technical guidance on laboratory techniques, influenza surveillance standards, influenza vaccine virus strains, and other pharmaceutical and non-pharmaceutical measures to mitigate influenzarelated morbidity and mortality.

\section{HISTORICAL PERSPECTIVE}

Ever since its inception, GISRS has played key roles in the response to 3 influenza pandemics (in 1957, 1968, and 2009), outbreaks of animal influenza viruses, and the occasional spill-over of these viruses into humans, also including other non-influenza events such as the SARS outbreak in 2002 and the current SARS-CoV-2 pandemic. These events have highlighted the important international coordinating role of WHO and GISRS in pandemic preparedness and response (7).

GISRS continues to monitor the influenza viruses circulating in animal populations that may be a risk to humans. Working closely with international animal health partners, such as the Food and Agriculture Organization (FAO) (http://www.fao.org) and the World Organisation for Animal Health (OIE) (8), GISRS works to detect and respond to the sporadic spill-over of animal influenza viruses into humans (zoonotic transmission events).

In 2011, WHO's Member States and Executive Board called upon WHO's Global Influenza Programme and GISRS to expand its work on pandemic preparedness and response with the adoption of the Pandemic Influenza Preparedness Framework for the Sharing of Influenza Viruses and Access to Vaccines and Other Benefits (PIP Framework). The objective of the framework was to improve influenza pandemic preparedness and response by improving and 
strengthening GISRS (9). With partners globally, the PIP Framework assists Member States with building national and international capacities to better detect and respond to inevitable future pandemics of influenza and beyond.

\section{ACHIEVEMENTS}

The achievements of GISRS and WHO's Global Influenza Programme since its inception, notably the timely response to the 2009 influenza pandemic, have been demonstrated and documented elsewhere ( 7 , 10-11). However, it is worth outlining how GISRS and associated influenza surveillance systems have also significantly contributed to the response to COVID-19 pandemic, caused by a non-influenza respiratory virus.

\section{National Influenza Centres were Crucial to Initial Laboratory Response During COVID-19}

National Influenza Centres (NICs) are the backbone of GISRS, collecting clinical specimens from sentinel and non-sentinel sources in their country, testing them for influenza and other respiratory viruses, performing preliminary analyses on influenza viruses detected, and sharing representative specimens or viruses with WHO Collaborating Centres (CCs) for advanced antigenic and genetic analyses. NICs are designated by national Ministries of Health and recognized by WHO. Given the capacities and expertise in these laboratories of the GISRS network, they became COVID-19 testing centres in many countries early in the SARS-CoV-2 pandemic. As of July 2020, approximately $85 \%$ of over 220 national COVID-19 reference or testing laboratories globally were either NICs or closely associated with GISRS (12).

\section{National Influenza Centres have Demonstrated Expertise of Virus Detection}

The first External Quality Assessment Project (EQAP) for SARS-CoV-2, which was critical to ensure national capacity of laboratory detection of the novel virus, was undertaken through the GISRS annual influenza EQAP mechanism, coordinated by WHO's GIP (13). A total of 233 laboratories (including 130 NICs) in 164 countries, areas, and territories participated, with the survey dispatched to labs from April to June 2020, despite disruptions caused by the pandemic. Overall, 94\% of laboratories received a $100 \%$ pass rate. Of the 233 laboratories that participated in the SARS-CoV-2 EQAP, 68\% $(159 / 233)$ also participated in the influenza EQAP in 2019 , indicating the substantial capacity for influenza testing that was utilized for testing during the COVID-19 response. Among laboratories that participated in the 2019 influenza EQAP, 94\% of the labs scored $100 \%$ on the 2020 SARS-CoV-2 EQA.

\section{Surveillance Data Generating and Sharing to Global Platforms}

Data is key to understanding the dynamics of and informing the response to seasonal epidemics and pandemics. A key component of GISRS is the global data platform FluMart, in place since 1997, where countries report data on samples processed for influenza, such as the number of positive samples and subtype, on a weekly basis to a dataset called FluNet. Outputs of the data are visible to the public on WHO's website (14). Since the emergence of SARS-CoV-2, WHO has requested that Member States regularly report counts of COVID-19 cases and deaths at different levels of aggregation for global situational awareness. FluMart formed the basis of the data collection platform for COVID-19 data (COVMart) since early in the pandemic. Globally, as of September 10, 2021, there have been 223,022,538 confirmed cases of COVID-19, including 4,602,882 deaths, reported to WHO through COVMart and displayed on the WHO COVID-19 dashboard (15).

\section{Genomic Sequencing and Genetic Sequence Data Sharing}

The sharing of virus genetic sequence data is essential, especially in the early stages of the response to an outbreak of a novel virus. Sequence data allows for the development of laboratory protocols to enable countries to detect and monitor novel viruses. GISAID (available at: https://www.gisaid.org/), which was launched in 2008 to enable rapid and open access to epidemic and pandemic influenza virus genetic data and associated clinical and geographical information through its EpiFlu platform, quickly became the host of the genetic sequence data of SARS-CoV-2. In fact, it published the first SARS-CoV-2 sequence data hours after it became available. GISAID has been an important partner of GISRS with confidence from 
countries for sharing their data through its data sharing mechanism. The development of the EpiCoV database and associated tools has been instrumental in the COVID-19 pandemic response. As of September 12, 2021, since the emergence of SARS-CoV-2, nearly 3.5 million complete genome sequences of SARS-CoV-2 have been uploaded to GISAID. About $80 \%$ of GISRS laboratories uploaded complete genome sequence data of SARS-CoV-2 to GISAID EpiCoV. In 2021, GISAID has also expanded to collect and share information on RSV, aside from influenza and SARS-CoV-2, though its EpiRSV data platform.

\section{LEVERAGING EXISTING SURVEILLANCE SYSTEMS FOR MONITORING COVID-19}

Sentinel surveillance is an efficient way to collect high-quality data in a timely manner systematically (using a consistent case definition and sampling strategy) and routinely from representatives of the population under surveillance so that the information gathered can be applied to the population or among subpopulations at higher risk of developing severe disease. Sentinel surveillance for COVID-19 using GISRS complements COVID-19 surveillance activities under the overall COVID-19 pandemic response. It is a cost-effective way to meet pandemic response objectives, including monitoring the geographic spread, intensity of transmission, and severity trends of community transmission of COVID-19 over time; understanding the risk factors for disease; systematically monitoring the genetic evolution of the COVID-19 virus; and assessing the impact on health systems. In addition, understanding the relative contribution of SARS-CoV-2 to the data captured in syndromic disease surveillance systems is critical to guide national responses and interpret the burden of disease caused by SARS-CoV-2 and influenza viruses. The data gathered can be used as a baseline for future evaluations of COVID-19 interventions such as vaccination, as has been already undertaken for influenza (12).

Since March of 2020, GISRS has incorporated SARS-CoV-2 into laboratory algorithms for testing specimens obtained from sentinel surveillance to monitor trends in co-circulation of the influenza and SARS-CoV-2 viruses and has provided guidance on the reporting of this information to FluNet, the global dataset managed by GIP (16). This integrated surveillance has been enhanced by the development, validation, and procurement of multiplex assays to test samples for influenza and SARS-CoV-2 simultaneously. In response to the recommendations of the 6th International Health Regulations (2005) (IHR) Emergency Committee for COVID-19, WHO encourages GISRS laboratories to sequence SARS$\mathrm{CoV}$-2-positive samples from its sentinel surveillance in a timely manner and share genetic sequence data with accompanying metadata through publicly accessible databases such as the GISAID EpiCoV database, with a focus on data quality over quantity (17).

\section{CURRENT CHALLENGES AND THREATS}

Much progress has been made on building capacities globally, regionally, and nationally to protect the world from influenza. However, in addition to the disruptions caused by the COVID-19 pandemic to livelihoods and health care systems, and the threat of misinformation, there are some challenges unique to influenza surveillance worth including here.

Although in most parts of the world, influenza activity has been lower than usual during the COVID-19 pandemic, an unusual epidemic can break out anytime. On the other hand, animal influenza viruses continue to sporadically infect humans, highlighting the continuing threat of zoonotic transmission and the need to remain vigilant for the unexpected and work with the One Health concept in mind (18). We also need to remain open to the possibility of another respiratory virus emerging and causing the next pandemic.

It is not clear when seasonal influenza activity will resume, and at what levels globally, but it is clear the surveillance systems need to be in place and functioning to quickly detect the return of influenza activity, alongside monitoring the trends postCOVID-19. In many countries, the current priority is understandably the response to the COVID-19 pandemic and exhaustive case finding and management efforts to control transmission. Contextspecific solutions to achieving the pandemic response objectives and the ongoing influenza surveillance objectives need to be put in place. The collecting and sharing of influenza data and viruses need to continue to ensure the world is as prepared as possible against influenza epidemic and pandemic threats. 


\section{FUTURE DEVELOPMENT}

With these challenges in mind, WHO has been developing a roadmap to further build GISRS into GISRS+: an enhanced network, built upon existing influenza infrastructure, to achieve integrated surveillance and response systems to influenza and a range of other respiratory viruses with epidemic or pandemic potential. The roadmap will address two main areas of work: 1) developing technical capacities; and 2) exploring coordination mechanism options for a GISRS+ system. The GISRS+, as envisaged with extensive input from GISRS members, countries and international agencies, will be a natural extension given the strength and successes the system had demonstrated over the past 70 years.

Acknowledgments: The GISRS network and all national and institutional public health professionals and all the generations of scientists throughout the world who have dedicated themselves to influenza surveillance, preparedness, and response, collaborating, generating and sharing of data; current and past WHO Influenza preparedness and Response, Global Influenza Programme and Pandemic Influenza Preparedness Framework staff and consultants working across the global, regional, and country levels.

doi: $10.46234 / \mathrm{ccdcw} 2021.226$

\# Corresponding author: Aspen Hammond, hammonda@who.int.

${ }^{1}$ Global Influenza Programme, World Health Organization, Geneva, Switzerland.

Submitted: September 30, 2021; Accepted: October 19, 2021

\section{REFERENCES}

1. Zhang WQ, Wood J. The global influenza surveillance and response system-65 years of building trust and sharing and a role model for global health security. Influenza Other Respir Viruses 2018;12(5):566. http://dx.doi.org/10.1111/irv.12548.

2. World Health Organization. Global influenza surveillance and response system (GISRS). https://www.who.int/initiatives/global-influenza- surveillance-and-response-system. [2021-9-20].

3. World Health Organization. FluNet. https://www.who.int/tools/flunet. [2021-9-20].

4. World Health Orgnization. FluID. https://www.who.int/teams/globalinfluenza-programme/surveillance-and-monitoring/fluid. [2021-9-26].

5. World Health Organization. RSV surveillance. https://www.who.int/ teams/global-influenza-programme/global-respiratory-syncytial-virussurveillance. [2021-9-26].

6. World Health Organization. Global influenza programme. https:// www.who.int/teams/global-influenza-programme. [2021-9-20].

7. Ziegler T, Mamahit A, Cox NJ. 65 years of influenza surveillance by a World Health Organization-coordinated global network. Influenza Other Respir Viruses 2018;12(5):558 - 65. http://dx.doi.org/10.1111/ irv. 12570 .

8. World Organisation for Animal Health (OIE). Responding to emergencies through preparedness and resilience. https://www.oie. int/en/home/. [2021-9-20].

9. World Health Organization. Pandemic influenza preparedness (PIP) framework. https://www.who.int/initiatives/pandemic-influenza-prepar edness-framework. [2021-9-20].

10. Hay AJ, McCauley JW. The WHO global influenza surveillance and response system (GISRS)-a future perspective. Influenza Other Respir Viruses 2018;12(5):551 - 7. http://dx.doi.org/10.1111/irv.12565.

11. World Health Organization. Implementation of the international health regulations (2005): report of the review committee on the functioning of the international health regulations (2005) in relation to pandemic (H1N1) 2009. 2011. http://apps.who.int/gb/ebwha/pdf_files/WHA64/ A64_10-en.pdf?ua=1. [2021-9-20].

12. World Health Organization. GISRS sentinel surveillance for COVID-19: frequently asked questions (FAQ). 2020. https://apps.who. int/iris/bitstream/handle/10665/333616/WHO-2019-nCoV-influenzaFAQ-2020.1-eng.pdf?sequence=1\&isAllowed=y. [2021-9-20] .

13. World Health Organization. Global influenza programme: external quality assessment project. https://www.who.int/teams/global-influenzaprogramme/laboratory-network/eqa-project. [2021-9-26].

14. World Health Organization. Influenza surveillance outputs. https:// www.who.int/teams/global-influenza-programme/surveillance-and-monitoring/ influenza-surveillance-outputs. [2021-9-26].

15. World Health Organization. WHO coronavirus (COVID-19) dashboard. https://covid19.who.int. [2021-9-26].

16. World Health Organization. Maintaining surveillance of influenza and monitoring SARS-CoV-2 adapting global influenza surveillance and response system (GISRS) and sentinel systems during the COVID-19 pandemic, interim guidance. 2020. https://apps.who.int/iris/rest/ bitstreams/1316069/retrieve. [2021-9-20].

17. World Health Organization. Operational considerations to expedite genomic sequencing component of GISRS surveillance of SARS-CoV2. 2021. https://apps.who.int/iris/rest/bitstreams/1332762/retrieve. [2021-9-20].

18. World Health Organization. Global influenza programme: risk assessment summaries of influenza at the human-animal interface. https://www.who.int/teams/global-influenza-programme/avianinfluenza. [2021-9-26]. 